\title{
Téoros
}

Revue de recherche en tourisme

\section{Intervention gouvernementale et tourisme régional}

\section{Réjean Beaudoin}

Volume 9, numéro 1, mars 1990

Plans et politiques touristiques

URI : https://id.erudit.org/iderudit/1080288ar

DOI : https://doi.org/10.7202/1080288ar

Aller au sommaire du numéro

Éditeur(s)

Université du Québec à Montréal

ISSN

0712-8657 (imprimé)

1923-2705 (numérique)

Découvrir la revue

Citer cet article

Beaudoin, R. (1990). Intervention gouvernementale et tourisme régional.

Téoros, 9(1), 18-20. https://doi.org/10.7202/1080288ar d'utilisation que vous pouvez consulter en ligne.

https://apropos.erudit.org/fr/usagers/politique-dutilisation/ 


\section{Intervention gouvernementale et tourisme régional}

Le phénomène du tourisme a sans contredit fortement progressé au cours des demières années et s'annonce à poursuivre cette lancée durant la décennie "90 et au-delà.

En effet, malgré une récession économique qui a marqué cette période, selon un document de Tourisme Canada', de 1975 a 1986 , les recettes touristiques ontaugmenté at un rythme annuel de $5,4 \%$ soit undes taux de croissance les plus élevés observés parmi les différents secteurs du commerce mondial:

Recettes touristiques internationales $5,4 \%$ Produit intérieur brut des

pays de l'OCDE 2,9\%

Exportations mondiales totales $\quad 3,5 \%$

Exportations mondiales

de produits agricoles

Exportations mondiales

de minerais

Exportations mondiales

de biens manufacturés

et pour l'avenir, les prévisionnistes les plus articulés extrapolent un taux de progression pouvant varier entre $3 \%$ et $6 \%$ annuellement d'ici 1995, assorti d'une vigueur probablement identique jusqu' 'à l'an 2000.

\section{Pour le Québec}

Depuis 1980, des indicateurs de performance de l'industrie touristique du Québec font ressortir les faits suivants:

- relativement au P.I.B. québécois, la part du tourisme diminue en passant de $3,2 \%$ (1980) a $2,5 \%(1987)$

- les recettes provenant des Québecois au Québec diminuent en passant à $41,2 \%$ (1980) pour aboutir à $34,3 \%$ (1986);

- le déficit touristique du Québec a doublé de 1980 à 1987 pour ravir 580 millions de dollars - ce déficit sera probablement de l'ordre de 700 millions en 1990;

- une stabilité relative des recettes touristiques globales en dollars constantsdurant lapériode 1980-1986.
Les grandes causes de cette stagnation apparaissent être la concurrence à l'échelle mondiale, une offre touristique québécoise manquant d'attraits forts et peu adaptés à la demande, la dispersion et la timidité des efforts de mise en marché, des moyens financiers plus limités par rapport à nos concurrents.

Ainsi, deux (2) defis s'adressent au Québec touristique:

- réaliser le plus rapidement possible le rattrapage quali quanii qui le caractérise:

- faire les efforts requis afin d'être à point, en l'an 2000, où le tourisme sera probablement la principale denrée des. exportations mondiales.

\section{Le tourisme et le développement régional}

Une région forte et prospère est celle qui a établi une économie diversifiée. A ce titre, un récent rapport ${ }^{2}$ publié par la Confédération des Caisses populaires et d'économie Desjardins du Québec soutient:

"En somme, plus une région s'appuie sur une base économique diversifiée, plus elle présente une meilleure croissance économique, de ce fait, un niveau de vie élevé non seulement sur le plan économique, mais aussi sur le plan social et culturel."

Le tourisme est un moyen de diversification économique. Sur ce plan, la Chambre de Commerce des Êtats-Unis (US Chamber of Commerce) déclare:

"Il y a trois (3) façons de développer l'économie d"un milieu: l'agriculture, l'industrie et le tourisme. Le tourisme est la façon la plus rapide et la plus facile des trois (3)."

Pour un milieu donné, l'activité touristique peut être soit une voie économique principale ou une source de revenus importants ou encore une entité économique d'appoint. A divers degrés, 1'industrie 
touristique peut contribuer positivement à la vie et à l'essor socio-économique d'une localité, d'une région, d'un pays.

\section{Le défi à venir}

La société se transforme et le Québec touristique doit suivre cette évolution: vieillissement de la population, les ménages à double revenus, les professionnels à revenus élevés (souvent célibataires), les touristes sont plus connaisseurs, plus instruits, plus raffinés... Cetté société du voyage accorde plus $\mathrm{d}^{\mathrm{*}}$ importance à la qualité du serviceet exige un rapport qualité/ prix conforme.

Afin de répondre à cette constante mutation et le secteur privé et le secteur public doivent faire leur part sur une base complémentaire, travailler en partenariat.

\section{L'intervention gouvernementale en région}

Les finances publiques se confondent avec l'activité financière de l'État et des autre collectivités publiques, elles engagent, pour citer Henry Laufenburger ${ }^{* i}$ une fraction plus ou moins importante des ressources nationales pour permettre aux collectivités d'accomplir leur tâche. ${ }^{7+3}$

La marge de manoeuvre de participation ou de contribution gouvernementale en région afin de favoriser l'expansion touristique qualitative et quantitative se situe entre l'approche zéro et l'approche étatique.

L'approche zéro a certainement uncaractère inéquitable, alors que l'approche étatique peut se justifier dans certains cas comme la création d'un parc national par exemple.

A travers le monde, l'assistance financière gouvernementale a l'industrie touristique prend différentes formes:

- achat d'obligations par le gouvemement remboursable sur une période de vingt (20) ans a un taux de $5 \%$ par année;

- subvention directe;

- prêt non-garanti;

- prise en charge des intérêts sur un emprunt;

- achat de terrains et location a long terme à un promoteur;

- crédit touristique sous diverses formes;

- exemptions d'impôt et de taxes;

- etc...
Autant l'imagination humaine peut être fertile, autant il peut $y$ avoir des idées impliquant l'intervention gouvernementale sur le plan touristique.

Comme le sujet est vaste et le contexte economique actuel fort réservé, ce texte se limitera à trois (3) éléments pouvant favoriser sćrieusement l'expansion du tourisme en région sur les plans de la concertation, du développement et de la publicité/promotion.

\section{La concertation}

La concertation régionale est la base originale de réussite en matière de tourisme. Plus elle est forte, plus il y a progression. Plus elle est faible, plus il y a régression.

En vertu de la Loi de proximité attitrée au tourisme, le touriste nord-américain se déplace sur une base régionale et interrégionale. Afin de capter le maximum d'impact, les intervenants doivent se concerter régionalement puis, selon les besoins, interrégionalement (l'approche produit).

Ainsi, l'État se doit de soutenir les organismes touristiques régionaux québécois (ATR) dans le but de maintenir et accentuer l'ascendance touristique du Québec sous toutes ses formes.

\section{Le développement}

Pour le développement, l'intervention gouvernementale en région devrait reposer sur deux (2) principes soit: le principe de base et le principe de complémentarité.

Le principe de base: les attraits et les activités permanents

Dans le monde du tourisme, ce sont les attraits et les activités (place, monument, hospitalite, etc.) qui donnent naissance, maintiennent ou renforcent les flux touristiques vers une région donnée. Ils sont l'ALPHA de la localisation touristique. Ce sont ni plus ni moins les matières premières qui invitent au voyage. Sans leur apport, il est quasi impossible à tout complexe touristique (tourist plant) de subsister.

On ne peut imaginer un ensemble d'hôtels et d'auberges sans attrait et activite; il y aurait peu de fréquentation et le site serait voué à une rapide désuétude.
Le comitét mixte de travail MTO/ATR sur le suivi des plans de développement touristique régionaux stipulait dans son rapport annuel présenté au ministère du Tourisme du Québec, le 17 novembre 1988:

"L'intervention de I'État devrait être canalisée vers la mise en oeuvred'attraits et d'activités aptes à générer un achalandage touristique et, par conséquent, des infrastructures de support."

Étant la ligne de départ ou d'amélioration de la fréquentation touristique, de par leur rôle, la miseen valeurd'attraitset d'activités représente un plus grand risque financier et. de mise en marché comparativement aux autres éléments de l'industrie du tourisme. Aussi, compte tenu de leur importance et de leurs effets d'entraînement, l'État devrait primordialement rendre l'investissement privé dans les attraits etactivités permanents aussi intéressant que $\mathrm{d}^{+}$autres investissements du monde ćconomique. Ce stade prioritaire acquis, le Québec touristique serait en mesure de vivre encore plus la maturation suivante:

- augmeniation du flux touristique dans les diverses régions touristiques québécoises;

- augmentation du rendement de beaucoup d'investissements actuels dans le secteur des services touristiques (hôtels, restaurants, boutiques);

- susciter de nouveaux investissements à plus faible risque dans le secteur des services touristiques;

- permetre au Québec d'effectuer le rattrapage qui le caractérise;

- permetre au Québec d'être à point, en l'an 2000 , où le tourisme sera la principale denrée des exportations mondiales.

Où il y a des attraits et des activités permanentes bien organisés, il y a des touristes et des services touristiques rentables. Où cette caractéristique est absente, il n"y a pas ou peu de touristes.

En somme, le principe de base à savoir l'intervention gouvernementale en région est cristallisée comme support d'appoint incitatif auprès du secteur privé en fonction des activités et attraits permanents représente une excellente orientation étatique pour la relance du tourisme québécois. 


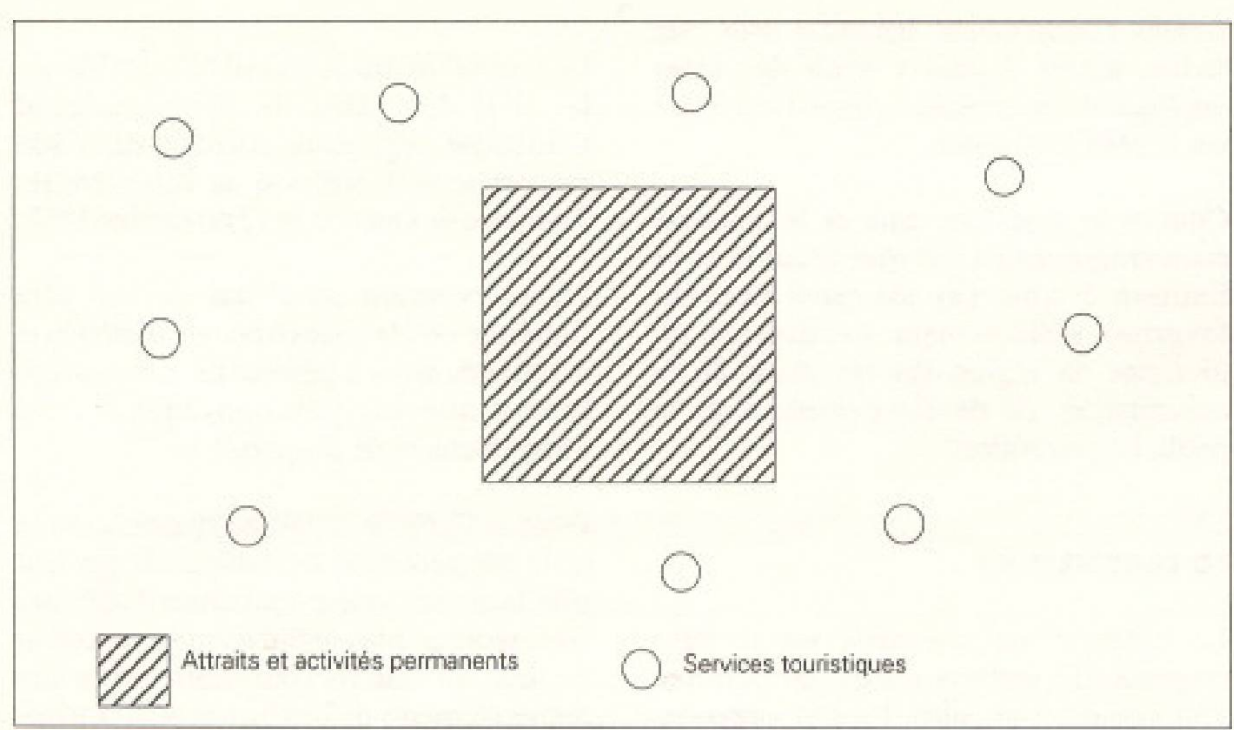

\section{Le principe complémentaire}

Ceprincipe est complémentaire au principe de base. Il est une approche circonstancielle modulée par région attitrée au secteur de l'hébergement.

Nous savons que les connaissances du monde doublent à tous les sept (7) ou dix (10) ans. A cette cadence, il est indubitable que l'art et les goûts reliés au voyage changent rapidement.

Il existe certains milieux régionaux québécois nantis d'attraits et d'activités permanents qui ont à faire face ou auront à faire face à une demande progressive, mais dont le secteur de l'hébergement commandent une amélioration rapide (la pourvoirie par exemple).

Aussi, pour ce type de situation localisée dans le temps et dans l'espace, une intervention gouvernementale incitative se révélerait justifiée et rentable. Elle serait ni plus ni moins une soupape ponctuelle permettant au secteur privé de s'ajuster rapidement, sur le plan hébergement, en regard $\mathrm{d}^{*}$ une nouvelle demande potentielle en pleine effervescence susceptible d'apporter d'importantes recettes touristiques pour les intervenants et le Quebec.

\section{La publicité et la promotion coopératives État/industrie}

L'intervention financière gouvernementale en matière de publicité et de promotion région, formée de la concertation, du développement prioritaire d'attraits et d'activités et de la publicité et de la promotion cooperatives Etat/INDUSTRIE chemine en partie depuis quelques années au Québec.

Toutefois, son application à son merite permettrait, sans aucun doute, une nouvelle vigueur montante de l'industrie touristique régionale et celle du milieu québécois dans son ensemble.

En somme, autant le tourisme régional que celui du Québec reposent sur la VITESSE avec laquelle ils sont en mesure de s'adapter aux changements de cette industrie afin de répondre, en tout temps, aux besoins de leurs diverses clientèles.

Tout le processus peut se résumer en un systèmed'action dynamique permanentque 1'on pourrait baptiser VICPPR soit:

VITESSE

vitesse d'information est une excellente voie pour le milieu régional. Chaque dollar gouvernemental attire des fonds du secteur privé pour constituer une synergie financière vivante dont les résultats ne peuvent qu'être probants.

Émettons 1'hypothèse que le ministère du Tourisme investit 250000 \$ dans lapublicité et la promotion coopératives d'une région et que les intervenants/ATR en injectent autant. Une enveloppe totale de 5000005 est ainsi formée.

Une telle politique, pour une région, permet une concertation étroite et concrète État/ INDUSTRIE, augmente les moyens de publicité et de promotion, multiplie la fréquence des actes publicitaires et promotionnels, assure une meilleure pénétration des marchés segmentés et amène plus d'intervenants à faire connaître directement leurs produits respectifs auprès des consonmateurs visés (versus la publicité institutionnelle).

De fait, plus les régions touristiques québécoises auront à leur disposition une enveloppe suffisante aux fins de publicité et de promotion coopératives, plus leur notoriété et celle du Québec grandiront.

\section{En terminant}

Tel que mentionné antérieurement, la nature du sujet laissait une place généreuse à différentes philosophies. Cette trilogie, en termes d'intervention gouvemementale en vitesse de concertation

vitesse de production

vitesse de promotion

vitesse de réservation

Et le cycle recommence!

\section{NOTES EXPLICATIVES}

(1)TOURISME CANADA, Les défis que pose le développement du produit touristique, Calgary. 1988.

(2) MONTOUA, Bruno, Profil des régions du Québec 1971-1987. La Conféderation des Caisses populaires et d'économie Desjardins du Québec, Lévis, 1988, p. \$8.

(3) Laufenburger, Henry. Théorie économique et psy1956, p. 5.

(4) Aujourd'hui, le comité mixte de travail MTO/ATR sur le développement touristique. chologique des finances publiques. Tome I, Peris, 